\title{
Participación y calidad de vida en familias de personas con discapacidad
}

\section{Participation and quality of life in families of persons with disabilities}

\author{
Daniela Vanessa Palma Arroyo ${ }^{1}$ \\ Jhoan Sebastián Zapata López² \\ Melania Satizabal Reyes ${ }^{3}$ \\ Pablo Esteban Roa Urrutia ${ }^{4}$
}

Recibido: 28 de enero 2016 • Enviado para modificación: 30 de junio de 2016 • Aceptado: 23 de julio de 2016

Palma, D., Zapata, J., Satizabal, M., \& Roa, P. (2016). Participación y calidad de vida en familias de personas con discapacidad. Revista Ocupación Humana, 16 (1), 19-31.

\begin{abstract}
Resumen: El estudio de la calidad de vida en familias de personas con discapacidad ha cobrado importancia desde un enfoque de capacidades. En esta categoría influye la comprensión de factores contextuales y de cómo estos facilitan la participación de las personas en su vida cotidiana. La investigación tuvo como objetivo caracterizar seis familias de personas con discapacidad de las zonas rural y urbana de Cali, en términos de calidad de vida familiar y participación. Se realizó un estudio cualitativo y se aplicaron la Escala de calidad de vida familiar para familias de personas con discapacidad, el cuestionario WHODAS 2.0 y una entrevista semiestructurada. Se concluye que la calidad de vida familiar en personas con discapacidad es subjetiva y multidimensional, y está mediada por las relaciones intrafamiliares y la decisión de lo que es importante o no para su satisfacción. La participación de las personas con discapacidad en sus familias depende de la relación entre las funciones corporales y su capacidad de aporte, acorde con las demandas de la actividad y el contexto. La multidimensionalidad de la calidad de vida condiciona su abordaje a factores objetivos y subjetivos de cada familia, y es influenciada por los contextos físico, cultural, social y político.
\end{abstract}

Palabras clave: calidad de vida, familia, personas con discapacidad.

Abstract: Research on quality of life in families of persons with disabilities has gained importance from an approach focused on capabilities. This category is influenced by the understanding of contextual factors and how these facilitate people's participation in their daily lives. The objective of this research was to characterize the family of six persons with disabilities in the rural and urban area of Santiago de Cali, in terms of quality of family life and participation. A qualitative study was conducted, using The family quality of life scale for families of people with disabilities, the questionnaire WHODAS 2.0 and a semi-structured interview. The quality of family life in persons with disabilities is subjective and multidimensional, and is mediated by intra-family relationships and the decision process of what is deemed important or not for their satisfaction. Family participation of disabled people depends on the direct relationship

\footnotetext{
${ }^{1}$ Terapeuta Ocupacional. Vicerrectoría de Bienestar Universitario, Universidad del Valle. Cali, Colombia. danielapalmaarroyo@gmail.com

${ }^{2}$ Terapeuta Ocupacional. Centro de Apoyos Educativos. Cali, Colombia. jhoanzapata_77@hotmail.com

${ }^{3}$ Terapeuta Ocupacional. Especialista en Desarrollo Comunitario. Maestrante en Sociología. Docente Escuela de Rehabilitación Humana, Universidad del Valle. Cali, Colombia.melania.satizabal@correounivalle.edu.co

${ }^{4}$ Terapeuta Ocupacional. Fundación Valle del Lili. Cali, Colombia. pabloroau@gmail.com
} 
between their bodily functions and their ability to contribute, according to the demands of the activity and the context. The multidimensionality of the quality of life conditions the way it is approached to objective and subjective factors in each family, influenced by the physical, cultural, social and political context.

Key words: quality of life, family, disabled persons.

\section{Introducción}

El tránsito que vive la discapacidad, de un paradigma de la rehabilitación a uno de la autonomía personal, ha conllevado a que su estudio no se centre solo en los asuntos del individuo y sus deficiencias, sino en los diferentes entornos y sistemas en los que interactúa (Díaz, 2009). La familia es el primer sistema en el que el ser humano se relaciona y en el que se generan los primeros vínculos con el entorno social, es considerada como "aquel sistema o conjunto organizado e interdependiente de personas en constante interacción, que se regula por unas reglas y por funciones dinámicas que existen entre sí y con el exterior" (Espinal, Gimeno \& González, 2004, p. 21).

La presencia de una persona con discapacidad en el hogar modifica o diversifica los procesos internos que en ella tienen lugar. De acuerdo con Manjarrés, León, Martínez y Gaitán (2013), "las familias que viven esta situación se ven expuestas a retos inimaginados, para los cuales no estaban preparadas, y requieren apoyo de la comunidad, de la sociedad en general y del Estado en particular" (p.23).

En este marco, el estudio de la calidad de vida en las familias de personas con discapacidad ha cobrado importancia. La tendencia frente al reconocimiento de la persona con discapacidad desde su naturaleza holística y la complejidad de sus relaciones en los diferentes contextos ha sido motivo de cuestionamiento, cuyos propósitos se extienden más allá de la identificación de variables que mejoren o no su calidad de vida, a la reflexión en torno a los apoyos que requiere el núcleo familiar y la forma como participan las personas con discapacidad en sus actividades cotidianas.

La calidad de vida familiar, desde un enfoque de las capacidades, permite a las familias desarrollar sus potencialidades en la búsqueda de resultados acordes con sus necesidades y expectativas. Es una categoría multidimensional, vinculada a la calidad de vida de la persona con discapacidad y a la influencia de los factores contextuales (Córdoba, Verdugo \& Gómez, 2011). Ahora bien, la comprensión de la discapacidad desde el paradigma de la autonomía personal hace énfasis en la importancia de la influencia de dichos factores; por tal razón, la participación de las personas con discapacidad y la configuración de la calidad de vida familiar están influenciadas por el contexto.

Lo anterior implica entender a la persona con discapacidad en interacción con sus entornos, en este caso el familiar; a su vez, requiere conocer el funcionamiento en términos de la ejecución de una tarea y la participación 
en las actividades cotidianas. Participar "es el acto de involucrarse en una situación vital" (OMS, 2001, p.131), razón por la cual en esta investigación se tuvo en cuenta tal elemento para comprender la calidad de vida familiar de las personas con discapacidad.

En consecuencia, este artículo expone la caracterización de seis familias de personas con discapacidad del municipio de Santiago de Cali, tres de las cuales se encontraban en zona ladera rural del corregimiento Los Andes y tres en zona ladera urbana de la Comuna 20 de la cabecera municipal. La caracterización ahondó en la relación existente entre la calidad de vida familiar y la participación de las personas con discapacidad en la realización de actividades cotidianas, teniendo en cuenta las diferencias contextuales de las zonas urbana y rural.

\section{Metodología}

La investigación se desarrolló bajo una perspectiva cualitativa, con enfoque de estudio de caso, a partir de una selección a conveniencia de la población. Participaron seis familias que contaban, al menos, con un integrante con discapacidad de tipo físico, sensorial, cognitivo leve o psicosocial; habitantes de la Comuna 20 y el corregimiento de Los Andes de Santiago de Cali.

La recolección de datos se llevó a cabo mediante la aplicación de la Escala de Calidad de Vida Familiar para Familias de Personas con Discapacidad (ECVF) (Verdugo, Córdoba \& Gómez, 2006), herramienta estandarizada, validada y de uso libre que fue propuesta por el equipo del Center Beach de la Universidad de Kansas. Esta escala consta de 41 ítems que se agrupan en cinco factores: interacción familiar, rol parental, salud y seguridad, recursos familiares y apoyo para las personas con discapacidad. Su aplicación se llevó a cabo durante la primera visita a las familias, cada ítem fue contestado en presencia de todos sus miembros.

Una vez diligenciada la ECVF se realizó el análisis en el Mapa de Calidad de Vida Familiar, herramienta complementaria de la escala e insumo fundamental que permite visibilizar el contraste entre el nivel de importancia y satisfacción de cada uno de los factores evaluados, estructurando gráficamente dichas intersecciones para su interpretación.

Ahora bien, al considerar la participación como una categoría importante de análisis, se aplicó el Cuestionario para la Evaluación de Discapacidades de la Organización Mundial de la Salud (WHODAS 2.0), herramienta que se integra dentro del sistema conceptual de la Clasificación Internacional del Funcionamiento, de la Discapacidad y de la Salud (CIF). Su versión en español (Vásquez, Herrera-Castañedo, Vásquez \& Gaite, 2006) es de uso libre y fue validada por la Unidad de Investigación en Psiquiatría de Cantabria, España.

EI WHODAS 2.0 fue administrado con el fin de entender las dificultades que pueden presentar las personas con discapacidad al desarrollar actividades cotidianas. Se indagó respecto a la independencia y participación al realizar actividades correspondientes a los dominios de: comprensión y comuni- 
cación, capacidad para moverse en el entorno, cuidado personal, relacionarse con otras personas, actividades de la vida diaria y participación social.

Se empleó la versión del WHODAS de 36 preguntas; fue administrada por el entrevistador y contestada por la persona con discapacidad. Durante su aplicación la persona debía pensar en las dificultades que ha presentado en la ejecución habitual de las actividades correspondientes a cada dominio durante los últimos treinta días, tomando en consideración el aumento del esfuerzo, el malestar o dolor, la lentitud y los cambios en el modo en que las realiza. Una vez diligenciado el cuestionario se procedió a realizar la suma de los ítems calificados de 1 a 5, según su nivel de dificultad. El análisis se llevó a cabo a través de la interpretación de tales resultados y en relación con los hallazgos derivados de la aplicación de la ECVF.

Por último, con el fin de indagar por aquellos aspectos contextuales no identificados con la aplicación de las pruebas antes descritas, se elaboró una entrevista general que fue administrada a las familias participantes. Esta se llevó a cabo en un tiempo aproximado de 45 minutos, posteriormente fue transcrita con el fin de aportar al análisis de las variables de los instrumentos empleados.

Consideraciones éticas. Según la Resolución 8430 de 1993 del Ministerio de Salud, este estudio fue considerado como una investigación con riesgo mínimo, teniendo en cuenta que no se realizaron intervenciones o modificaciones intencionadas a los participantes. Las personas recibieron infor- mación previa a la ejecución del proyecto, se les explicaron los objetivos, la metodología y el tiempo de ejecución; así mismo, firmaron consentimientos informados que garantizaban su participación voluntaria y la no incurrencia en gastos o compensaciones económicas. No se hizo registro fotográfico ni fílmico. En el texto se omiten los nombres reales de los participantes, estos fueron reemplazados por seudónimos. El estudio contó con el aval del Comité de Ética Humana de la Universidad del Valle, mediante el Acta de aprobación No. 18-014 de 2015.

\section{Resultados}

\section{Familias participantes}

La primera de las tres familias de la zona urbana está compuesta por siete personas, conviven con un integrante con discapacidad de 20 años de edad, quien perdió movilidad en sus brazos y piernas desde la infancia. El nivel de estudios alcanzado por la mayoría de sus integrantes es la primaria básica; excepto dos personas, quienes nunca han ingresado a un sistema educativo regular. El sustento económico del hogar está a cargo de una de sus integrantes, quien devenga un ingreso mensual de $\$ 644.350$, equivalentes a un salario mínimo legal vigente 2015 (SMLV).

La siguiente familia está compuesta por dos mujeres, una de ellas tiene discapacidad física severa. La persona con discapacidad refiere que es madre de una joven de 15 años quien no vive con ella, es habitante de calle y consume sustancias psicoactivas. El ingreso mensual de la familia es superior a un SMLV 2015, entre una pensión 
por invalidez y el alquiler de parte de la vivienda; sin embargo, indican que el dinero que ingresa mensualmente no logra cubrir los gastos cotidianos y las necesidades de la persona con discapacidad.

La tercera familia de la zona urbana está compuesta por cuatro personas, dos de ellas con discapacidad; la primera es una mujer con epilepsia y obesidad, la otra, un hombre con esquizofrenia. El domicilio está ubicado en la parte alta de Siloé ${ }^{5}$, a donde se accede por medio de vehículos particulares. El sustento del hogar está dado por la venta de dulces y almuerzos; sus ingresos son inferiores a un SMLV 2015.

Las tres familias indican situaciones que consideran inseguras en el sector, como el consumo de sustancias alucinógenas en las canchas deportivas, hurtos, conflictos entre pandillas, entre otras. Ello limita su participación en actividades de la comunidad.

Por otra parte, las tres familias de la zona rural se ubican en el corregimiento Los Andes. La primera se compone por seis personas, una de ellas tiene discapacidad auditiva congénita, se desempeña como agricultor y aporta económicamente para el sustento del hogar junto a dos integrantes más. Logran un ingreso mensual correspondiente a un SMLV 2015.

La siguiente familia la integran seis personas, una pareja de adultos mayores, uno de ellos tiene enfermedad de Parkinson, tres adultos -hijos de la pareja- y una nieta adolescente. El domicilio en el que viven se encuentra construido de forma artesanal, en bahareque $^{6}$, sobre un terreno con desniveles que dificulta el desplazamiento a la persona con discapacidad. La pareja indica que únicamente uno de los hijos aporta al sustento del hogar, con ingresos mensuales inferiores a un SMLV 2015 para cubrir las necesidades del núcleo.

Por último, la tercera familia de la zona rural se compone de cuatro personas, una de ellas se encuentra en cama desde hace cinco años, como consecuencia de su avanzada edad y su condición de salud relacionada con demencia senil, hipertensión y limitaciones motoras; se le dificulta participar de actividades realizadas dentro del hogar y al exterior de este. La persona con discapacidad vive con tres de sus hijos, quienes se desempeñan como agricultores y devengan una suma inferior a un SMLV 2015.

Es importante señalar que en el corregimiento Los Andes, por hacer parte de una zona declarada parque natural, se prohíbe la construcción y remodelación de viviendas, además se restringe el cultivo de alimentos con fines de consumo o sustento económico. Las tres familias manifiestan inconformidad por no tener documentos de propiedad de los terrenos en los que viven.

\section{Calidad de vida en las familias de personas con discapacidad}

A partir del análisis realizado con el Mapa de calidad de vida se encuentra

\footnotetext{
${ }^{5}$ Siloé es uno de los barrios que integra la Comuna 20 del Municipio de Santiago de Cali.

${ }^{6}$ Construcción hecha con barro, paja, hierba seca y boñiga.
} 
que, para las seis familias participantes, la calidad de vida se reflejaría en la capacidad de acceder a servicios de salud y a la satisfacción de las necesidades básicas. Sin embargo, es representativo que para las familias que habitan la zona urbana la calidad de vida se centra principalmente en el acceso que tiene su familiar con discapacidad a bienes y servicios, mientras para las personas que habitan la zona rural dicho acceso debe abarcar a todos los integrantes de la familia.

Adicionalmente, en el corregimiento Los Andes el poder desempeñar actividades significativas y que han sido trasmitidas de generación a generación, como labrar la tierra, influye en la concepción de la calidad de vida que tienen las familias. Esto lo refiere una de las participantes:

"¿Sabe qué se me viene a la cabeza? Mantener la finca llena de cultivo, eso es para mí calidad de vida" (Cármen, 69 años, esposa de una persona con discapacidad. Corregimiento Los Andes).

Calidad de vida es además contar con vías de acceso en buenas condiciones, tener la libertad de desplazarse y de acceder a un servicio de salud cuando las condiciones así lo demanden. Al respecto, uno de los participantes refiere:

"¿Calidad de vida? Primero la salud, que aquí se dificulta mucho porque hay que pegar para la ciudad; aquí por lo menos no hay droguería, entonces hay que ir a Cali a conseguir los medicamentos" (Gonzalo, 62 años, hijo de una persona con discapacidad. Corregimiento Los Andes).

Del mismo modo, indican que calidad de vida es la oportunidad de hacer reformas en sus casas, lo cual está restringido por la condición de parque natural del corregimiento y por las circunstancias culturales, políticas, legales y sociales que ello implica ${ }^{7}$. Los campesinos de la zona y quienes allí habitan desde hace más de cien años se ven limitados en el uso de sus suelos para la producción de cosechas y cultivos; adicional a ello, sus casas, construidas artesanalmente, cada vez están más deterioradas por las condiciones climáticas del sector, y aun cuando ponen en riesgo sus vidas, la resolución no permite su modificación. Estas situaciones alteran su calidad de vida:

"No dejan construir, no dejan trabajar, no dejan nada. iCaramba! Y el gobierno jamás nos tomó en cuenta" (Carlos, 40 años, hijo de una persona con discapacidad. Corregimiento Los Andes).

"Esta tierra es de nosotros. La dejaron los abuelos, son tierras con más de cien años. Es injusto que no nos dejen reformar ellos" (Cármen, 69 años, esposa de una persona con discapacidad. Corregimiento Los Andes).

Factor Interacción familiar. Es común que los integrantes de las familias presenten relaciones de apoyo y cuidado dentro de su entorno próximo, y que busquen el logro del bienestar de la persona con discapacidad. Para el caso de quienes habitan en la zona urbana,

\footnotetext{
${ }^{7}$ Resolución 092 de 1968 expedida por el Instituto Colombiano de la Reforma Agraria - Ministerio de Agricultura. Por la cual "se reserva y declara Parques Nacionales Naturales a la zona conocida como Farallones de Cali" (p. 1), se sustenta la protección y conservación de los ecosistemas boscosos de Los Andes, que, desde principios del presente siglo, se encuentran respaldados por dichas acciones legales.
} 
sus esfuerzos se centran en la satisfacción de las necesidades de esta última:

"Siempre pienso en lo que tiene que ver con la niña, que pueda caminar, que la familia pueda verla caminar y ser feliz. Si ella está bien, todos estamos bien" (Mercedes, 49 años, madre de una persona con discapacidad. Comuna 20).

Sin embargo, quienes cumplen el rol de cuidadores han dejado de lado actividades significativas y de interés, de tal forma que han depositado la esperanza en un mejor futuro:

"Yo no tengo nada más que hacer en la casa que cuidarla a ella, y me siento feliz de hacerlo. Mi abuela desde pequeña me enseñó a cuidarla, porque nunca le ha gustado que me vaya para la calle; cuidarla a ella es mi trabajo, solo eso" (Valery, 27 años, hermana de una persona con discapacidad. Comuna 20).

Otra de las cuidadoras indica que limita su participación en actividades de interés para brindarle asistencia y protección a su familiar:

"No acostumbramos a ir a misa cada domingo porque yo tengo que cuidar a mi hija, y pues llevarla a la misa me queda muy difícil" (Miriam, 49 años, madre de una persona con discapacidad. Comuna 20).

En lo concerniente al corregimiento Los Andes, se hace notoria la organización y la distribución de los roles que han llevado a cabo las familias; acciones enmarcadas en el apoyo en las tareas del hogar y en el cuidado de la persona con discapacidad. Esto favorece el desarrollo de un proyec- to de vida individual y familiar. Así lo indica uno de los participantes de la zona rural:

"Aquí todos tenemos responsabilidades... mi hermano mantiene más al lado de ella, luego le toca que ir a Cali a conseguir las cosas, las frutas; luego pues yo lo remplazo. Y si no está él, pues yo hago las obligaciones, y así... cada uno aquí tenemos como una especie de responsabilidad" (Raúl, 58 años, hijo de una persona con discapacidad. Corregimiento Los Andes).

Factores Salud y seguridad - Apoyo a la persona con discapacidad. Estos se relacionan con la salud física y mental de las familias, la prestación de servicios y las condiciones de sus contextos físico y de vivienda. Es importante resaltar que, tanto en los sectores urbanos como en la zona rural, las familias definen como insuficiente la cobertura de su sistema de salud, siendo reiterativa la inconformidad al no obtener apoyo de entidades que conozcan las necesidades individuales de las familias y den el seguimiento y la continuidad pertinentes a los procesos emprendidos.

De igual forma, el acompañamiento de profesionales de la salud es escaso, incluso en el análisis de los diagnósticos; este aspecto es importante para estas familias, permeadas por un modelo biomédico-rehabilitador en el que la discapacidad es entendida como un problema personal, causado directamente por una enfermedad o traumatismo. Como consecuencia, en la mayoría de los casos se desconocen la etiología y el curso de la enfermedad, lo que genera falsas expectativas: 
"A mí el médico me dijo que eso era una enfermedad genética, pero vea, en la casa nadie sufre de eso. A ella me le hicieron esa terapia para la voz, la que le movían los pies y la que le ponía a jugar, pero ese Hospital Departamental dijo que ya era suficiente, no mejoró" (Josefina, 78 años, abuela de una persona con discapacidad. Comuna 20).

Es necesario destacar que para los habitantes de la zona rural estas situaciones son más críticas; las barreras contextuales y sociales del entorno, entre las cuales se encuentran la normativa que rige al sector y las condiciones geográficas y arquitectónicas del corregimiento, dificultan el acceso a los servicios de salud.

La relación entre el tipo de discapacidad y el nivel de independencia también guarda relación con el acceso a los servicios de salud. Para las personas que habitan la Comuna 20 es difícil llegar hasta una vía principal de la ciudad que las conduzca a sus centros de salud, sin embargo, cuentan con un medio de transporte que las puede acercar hasta sus destinos sin necesidad de desplazarse mucho desde sus viviendas. Por su parte, las familias que habitan el corregimiento Los Andes deben recorrer trochas para abordar el medio de transporte, que tras 45 minutos de recorrido, las acerca al casco urbano. El acceso a los servicios de salud desde la zona ladera rural resulta entonces más difícil, como consecuencia de las condiciones de las personas con discapacidad y las características físicas del contexto.

Para finalizar, las condiciones políticas y del sistema de salud siguen siendo barreras para las familias de zona ladera, especialmente en el contexto rural. El acceso y la atención en los servicios no logran ser integrales y autosuficientes, dadas la escasas inversiones que realizan las instituciones públicas para proporcionar servicios de salud completos y especializados en comunidades dispersas como esta, condicionadas además como parque natural.

\section{Participación de las personas con discapacidad}

Dominio Comprensión y comunicación. Para las personas de ambas zonas es influyente, al momento de asignar responsabilidades dentro de sus hogares, la capacidad de sus familiares para comprender, analizar, evocar información y brindar soluciones. Los participantes que presentan dificultades en sus funciones cognitivas ven limitadas sus posibilidades de aportar en la toma de decisiones o en el cumplimiento de tareas. Ello deja en evidencia la restricción generada por las características de la discapacidad, en la que las demandas del contexto no son consecuentes con la capacidad de aporte de las personas.

Contrario a esto, aunque las características de la discapacidad física de una persona limitan su vinculación directa en algunas tareas del hogar, el hecho de que su madre reconozca la capacidad cognitiva que tiene hace posible su participación mediante la planificación y estructuración de actividades; ello le permite aumentar su capacidad de aporte, por tanto, de brindar apoyo a su cuidadora:

"La niña no hace ninguna actividad dentro de la casa porque le queda 
difícil, a duras penas mueve los ojos, mueve algo del cuello, habla pasito y ya. Lo que sí suele hacer es que me recuerda cosas que debo hacer, porque se me olvidan, ella tiene muy buena memoria" (Miriam, 49 años, madre de una persona con discapacidad. Comuna 20).

Otro participante, aunque no habla, conserva el funcionamiento de los procesos vinculados con la compresión y el análisis de información, y tiene la capacidad de comunicarse a través del uso de señas; ello hace posible que se le asignen responsabilidades dentro del hogar:

"Él arregla su pieza, barre, acomoda su moto, y cuando lo llaman a trabajar, sale a recoger leña" (Fernando, 62 años, padre de una persona con discapacidad. Corregimiento Los Andes).

Caso contrario vive otra participante, quien por sus limitaciones motoras y la falta de lenguaje oral y corporal, tiene dificultades para intercambiar información con las personas que la rodean y para participar en actividades significativas, y no le son asignadas responsabilidades en su familia:

"No, ella no tiene ninguna responsabilidad. Ella no tiene esas habilidades. Nosotros la bañamos y la vestimos, todo" (Mercedes, 49 años; madre de una persona con discapacidad. Comuna 20).

\section{Dominio Capacidad de la persona para moverse en su alrededor. Abar-} ca las categorías: ponerse de pie, cambiar de posición corporal, moverse dentro del hogar, salir del hogar y desplazarse. En este dominio los participantes con discapacidad física indican mayores dificultades para la ejecución independiente de actividades cotidianas. Esta situación se intensifica cuando el contexto físico y social no brinda los elementos facilitadores requeridos para que cada persona participe plenamente.

Dominio Cuidado personal. Se identifican mayores demandas de apoyo en la medida que aumentan las dificultades de las personas con discapacidad para moverse. Cuatro de los seis participantes requieren de asistencia al realizar actividades como lavarse el cuerpo, vestirse y alimentarse; esto implica un acompañamiento constante de los familiares para la satisfacción de sus necesidades. Diferente a ellos, dos participantes realizan las actividades de autocuidado de forma independiente.

Otro factor relevante es el aspecto contextual, en el cual cobra nuevamente importancia el ambiente físico como barrera para el desarrollo de actividades. Al indagar al respecto a las diferentes familias, en el contexto rural emergen respuestas relacionadas con el acceso al territorio:

"Aquí la gente intenta pavimentar y es otro problema con esos malnacidos. No se puede hacer nada por ser parque natural" (Carlos, 40 años, hijo de una persona con discapacidad. Corregimiento Los Andes).

"Hay instituciones que no quieren que pavimenten, aunque eso ya estaba planeado" (Gonzalo, 62 años, hijo de una persona con discapacidad. Corregimiento Los Andes).

De igual forma, en la zona urbana los participantes refieren la presencia de 
barreras que restringen la participación de las familias y el acceso a servicios en diversas situaciones:

"La guala ${ }^{8}$ pasa muy llena, los MIOS son muy lentos y costosos, los taxis no suben, las ambulancias no llegan" (Mercedes, 49 años, madre de una persona con discapacidad. Comuna 20).

\section{Dominio Relacionarse con otras per-} sonas. Se identifica una estrecha relación entre las características de las personas con discapacidad y su participación en actividades del hogar y del contexto social. Estos aspectos se encuentran influenciados por la percepción y la representación social en torno a la discapacidad, el escaso reconocimiento de sus habilidades y las pocas oportunidades que les brindan sus sujetos próximos.

\section{Discusión}

Velásquez, Vilma, López, López y Cataño (2011) indican que:

El círculo entre la discapacidad y la pobreza es más recurrente en los hogares que tienen menos ingresos. A su vez, la discapacidad incrementa los riesgos de pobreza porque aparta a las personas de posibilidades para generar ingresos y se requiere de altos gastos económicos de su familia (p. 123).

Lo manifestado anteriormente se relaciona con los resultados de la presente investigación, en la que se identifica que las familias participantes presentan carencias significativas en sus recursos económicos, tienen pocas facilidades que les permitan acceder a programas y respuestas institucionales para cubrir sus necesidades básicas en cuanto a servicios de salud, trabajo y educación. Lo anterior, sumado a la discapacidad, representa condiciones de vulnerabilidad que limitan el desarrollo de procesos, el cumplimiento de expectativas y aspiraciones, y el acceso a las oportunidades que desean.

Estas familias además reclaman acciones por parte de la sociedad para la satisfacción de sus necesidades. Al reflexionar en torno a componentes objetivos de la calidad de vida, como la satisfacción de las necesidades básicas relacionadas con el acceso a servicios de salud, educación, seguridad y transporte, se identifica que en su mayoría se encuentran insatisfechos. Como refiere Barnes (2010), "los costes extraordinarios derivados de la discapacidad y de las barreras psicológicas, físicas y sociales a las que se enfrentan las personas con discapacidad contribuyen a su pobreza, falta de libertad y vulneración de derechos humanos" (p.1). Este hallazgo refleja la influencia directa de las características físicas, culturales, económicas, sociales y políticas propias del contexto de la zona ladera, un extenso territorio al lado del casco urbano, olvidado por programas, políticas y acciones de bienestar social.

\footnotetext{
${ }^{8}$ Las gualas o jeeps son vehículos tipo campero, modelos sesenta o setenta, con capacidad para más de diez personas. Se encuentran asociados como prestadores de servicios de transporte informal en zonas de ladera del municipio de Santiago de Cali.

${ }^{9}$ El Masivo Integrado de Occidente (MIO) es el sistema integrado de transporte de la ciudad de Santiago de Cali. Es operado por buses articulados desde el 1 de marzo de 2009. Cuenta con rutas troncales, padrones y alimentadoras para la movilidad de sus usuarios.
} 
Se destaca además que para las familias de la zona rural estas situaciones son más críticas, debido a las barreras del entorno que aumentan como consecuencia de las regulaciones dadas por la condición de parque natural del corregimiento. Esta situación, sumada a la falta de reconocimiento de la misma población en torno al ejercicio de sus derechos como ciudadanos, restringe su participación en actividades productivas significativas, propias de su historia ocupacional, como es labrar la tierra. Por lo tanto, es fundamental apoyar y fortalecer a las familias en su interacción con formas de organización institucional, social y cultural que les permitan obtener respuestas a sus requerimientos como familias y comunidad.

Como se ha mencionado, haciendo referencia a Manjarrés (2012), las dinámicas en el hogar ante la llegada de una persona con discapacidad requieren de una flexibilización en los roles y responsabilidades; además, las respuestas que se brindan están estrechamente ligadas a las creencias y representaciones que se tienen de la discapacidad. En las familias de la zona urbana se tiende a asumir un rol de cuidado, atención y asistencia, asociándose la calidad de vida a las posibilidades de participación en su contexto que tenga la persona con discapacidad; incluso quienes desempeñan el rol de cuidador dejan a un lado actividades que para ellos son significativas. Por el contrario, en la zona rural los integrantes de los hogares han distribuido responsabilidades de tal forma que les permite brindar cuidado y protección a su familiar con discapacidad, al tiempo que desarrollan sus proyectos de vida.
Otro aspecto a resaltar es la representación que estas familias tienen acerca de la discapacidad. Se reconoce que conviven con una visión centrada en la persona como lo es, retomando a Brogna (2009), la médico-reparadora. Así, para las familias participantes la situación de discapacidad que se presenta está puesta en el déficit, y la participación se relaciona con el grado de limitación; por tanto, a mayores dificultades de tipo motor, menor independencia en la realización de actividades cotidianas (autocuidado, productivas, ocio y tiempo libre) y mayor necesidad de asistencia. Del mismo modo, al presentar limitaciones para comprender, analizar, brindar soluciones y procesar información, se restringe la capacidad de la persona con discapacidad para aportar a la toma de decisiones dentro del hogar.

\section{Conclusiones}

Teniendo en cuenta los hallazgos se puede concluir que el concepto de calidad de vida familiar es esencialmente subjetivo y multidimensional. La calidad de vida no se asocia solamente con el acceso a los servicios de salud y tampoco se restringe a las características de una persona, en este caso, con discapacidad. Por este motivo, es necesario llevar a cabo un análisis integral y exhaustivo que dé cuenta de las necesidades de las familias, de las acciones que se ejecutan en respuesta a sus representaciones de la discapacidad, de su participación en actividades significativas y del desarrollo de su vida familiar.

Al analizar la participación de las personas con discapacidad en activida- 
des cotidianas y la percepción acerca de la calidad de vida familiar es necesario tener en cuenta la influencia que ejerce el contexto físico, cultural, social y político donde se desarrollan sus vidas, debido a que estos factores pueden actuar como barreras o facilitadores en las prácticas que se efectúan al interior de la familia y en la comunidad.

Se hace evidente además que la distribución de roles y responsabilidades de forma equitativa al interior de la familia debe ser un factor que posibilite la participación y la autorealización de cada uno de sus integrantes, de manera que les sea posible responder a las demandas propias y a aquellas generadas por la condición de discapacidad de su familiar. Por tanto, es necesario avanzar en el estudio de las dinámicas familiares que en zonas rurales permiten una mejor distribución de roles y posibilitan la atención de la persona con discapacidad sin que ello implique dejar de participar en actividades cotidianas y significativas.

Por último, se identifica que el cubrimiento de las demandas básicas y la atención a las particularidades de las familias desde aspectos físicos, sociales, culturales y políticos posibilita la garantía de los derechos humanos y el reconocimiento del potencial y la capacidad de aporte que tienen las personas con discapacidad dentro de su contexto familiar y comunitario.

\section{Referencias}

Barnes, C. (2010). Discapacidad, política y pobreza en el contexto del "Mundo Mayoritario". Política y Sociedad, 47(1), 11-25.
Brogna, P. (2009). Visiones y revisiones de la Discapacidad. México: Fondo de Cultura Económica.

Córdoba, L., Verdugo, M.A., \& Gómez, J. (2011). Escala de calidad de vida familiar para familias de personas con discapacidad. Manual. España: Universidad de Salamanca.

Díaz, E. (2009). Reflexiones epistemológicas para una sociología de la discapacidad. Revista sociológica de pensamiento crítiCO, 3(2), 85-99.

Espinal, I., Gimeno, A. \& González, F. (2004). El enfoque sistémico en los estudios sobre la familia. Revista Internacional de Sistemas, 14 (2004-2006), 21-34.

Instituto Colombiano de la Reforma Agraria. (1968). Resolución 092 del 15 de julio 1968. Bogotá: Ministerio de Agricultura. Recuperado de: https://www.parquesnacionales.gov.co/PNN/portel/libreria/pdf/ Resolucion092de1968Farallones.pdf

Manjarrés, D., León, E. Y., Martínez, R., \& Gaitán, A. (2013). Crianza y discapacidad: una visión desde las vivencias y relatos de las familias en varios lugares de Colombia. Bogotá: Universidad Pedagógica Nacional.

Manjarrés, D. (2012). Apoyo y fortalecimiento a familias para la crianza de niños con discapacidad. Horizontes Pedagógicos, 14(1), 97-118.

Organización Mundial de la Salud (2001). Clasificación Internacional del Funcionamiento, de la Discapacidad y de la Salud - CIF. Ginebra, Suiza: Ministerio de Trabajo y Asuntos Sociales. Secretaría de Estado de Servicios Sociales, Familias y Discapacidad. Instituto de Mayores y Servicios Sociales (IMSERSO).

Vázquez, J. L., Herrera-Castañedo, S., Vázquez, E., \& Gaite, L. (2006). Cuestionario para la evaluación de la discapacidad WHODAS II. Versión en lengua española del World Health Organization Disability Assessment Schedule. Ministerio de Trabajo y Asuntos Sociales. Madrid. 
Velásquez, G., Vilma, F., López, L., López, H. \& Cataño, N. (2011). Tejido de significados en la adversidad: discapacidad, pobreza y vejez. Revista Hacia la Promoción de la Salud, 16(2), 121-131.
Verdugo, M., Córdoba, L. \& Gómez, J. (2006). Adaptación y validación al español de la escala de calidad de vida familiar. Revista Española sobre Discapacidad Intelectual, 37, 41-48. 